\title{
Thermodynamics of non-interacting Bosons in low-dimensional potentials
}

\author{
Gert-Ludwig IngOLD ${ }^{1}$ and Astrid LAMBrecht ${ }^{2}($ H) \\ 1 Institut für Physik, Universität Augsburg, Memminger Str. 6, D-86135 Augsburg, \\ Germany \\ ${ }^{2}$ Max-Planck-Institut für Quantenoptik, Hans-Kopfermann-Str. 1, D-85748 Garching, \\ Germany
}

PACS. 03.75Fi - Phase coherent atomic ensemble (Bose condensation).

PACS. 05.30Jp - Boson systems.

PACS. 64.60-i - General studies of phase transitions.

\begin{abstract}
On the basis of a macroscopic ground state population it was argued recently that Bose-Einstein condensation should occur in a one-dimensional harmonic potential. We examine this situation by drawing analogies to Bosons in a two-dimensional box, where the thermodynamic limit is well-defined. We show that in both systems although the ground state populations show sharp onsets at the critical temperature, the behaviour of the specific heat is analytic, which proves the absence of a phase transition in these systems.
\end{abstract}

The experimental study of ultracold trapped Bose gases [1, 2, 3] has revived the interest in Bose-Einstein condensation in the regime of weak or even vanishing interaction. In these experiments the atoms are confined by external forces, which might be modelled by a threedimensional harmonic potential. Recently it was suggested on the basis of results for the ground state population that in an effectively one-dimensional potential Bose-Einstein condensation should occur [4]. In the present paper we place the discussion of non-interacting Bosons in a one-dimensional harmonic potential into a larger framework by drawing analogies to particles in a two-dimensional box with infinite walls, a system which has, in contrast to the former one, the advantage that a well-defined thermodynamic limit exists. Within this framework we address the conceptual problem whether a macroscopic ground state population is a sufficient indicator for Bose-Einstein condensation to appear or if in contrast, thermodynamic quantities have to be considered. In addition to the ground state population we study in particular the specific heat, calculated with a continuous density of states, which is permissible for large particle numbers. The result is confirmed by an analysis based on the discrete level structure.

Bose-Einstein condensation [5] is usually described within the grandcanonical ensemble

$\left({ }^{*}\right)$ Present and permanent address: Laboratoire Kastler Brossel, Université Pierre et Marie Curie, Ecole Normale Supérieure, Centre National de Recherche Scientifique, 4 place Jussieu, BP74, F-75252 Paris Cedex 05, France

Typeset using EURO-IAT $\mathrm{E}_{\mathrm{E}} \mathrm{X}$ 
where the average number of particles is given by

$$
N=\int_{0}^{\infty} \mathrm{d} E \rho(E) \frac{z}{\exp (\beta E)-z} .
$$

Here, $z=\exp (\beta \mu)$ is the fugacity with $\beta=1 / k_{B} T$ and the chemical potential $\mu$. The energy scale is chosen such that the minimum value of the $d$-dimensional external potential $U(\mathbf{r})$ equals zero. Then the classical density of states for particles of mass $m$

$$
\rho(E)=\frac{1}{\Gamma(d / 2)}\left(\frac{m}{2 \pi \hbar^{2}}\right)^{d / 2} \int \mathrm{d} V_{\mathrm{cl}}[E-U(\mathbf{r})]^{(d-2) / 2},
$$

where the integration has to be taken over the classically accessible region, vanishes for negative energies. If $\rho(E) \rightarrow 0$ for small energies, $N$ reaches a finite maximum even for the maximum value of the fugacity $z=1$. Additional particles are then found in the ground state which is not included in the density of states (2). For a $d$-dimensional harmonic potential the density of states varies like $E^{d-1}$ and therefore Bose-Einstein condensation is only expected for $d=2$ and higher [6, 7].

This approach has been criticized by various authors. For the three-dimensional harmonic potential it was shown that corrections to the density of states (2) lead to corrections to the Bose temperature, which are of order $N^{-1 / 3}$ [8]. Other work [9, 10, 11, 12, 13] has addressed the question whether there are effects due to the discreteness of the energy levels which is not included in (2). In this context, it was recently claimed that Bose-Einstein condensation can be found even in the case of a one-dimensional harmonic potential 顿.

The argument given by Ketterle and van Druten is as follows. The relative ground state occupation $N_{0} / N$ is calculated by taking into account the discreteness of the energy levels. As an example, we show in fig. 1a results for particle numbers varying between $N=10^{2}$ and $N=10^{7}$ particles. The temperature is scaled with a critical temperature determined by 纯

$$
N=\frac{k_{B} T_{c}}{\hbar \omega} \ln \left(\frac{2 k_{B} T_{c}}{\hbar \omega}\right),
$$

where $\hbar \omega$ is the energy level spacing. It can be seen that with increasing number of particles $N$, the crossover between finite and vanishing ground state occupation sharpens. This behaviour is similar to the three-dimensional case, where Bose-Einstein condensation occurs, and it is thus concluded that there is condensation even in one dimension.

However, in a one-dimensional harmonic potential with fixed frequency $\omega$ the critical temperature $T_{c}$ diverges in the limit $N \rightarrow \infty$. It is therefore not obvious how a phase transition in such a system may be defined. Furthermore, a rescaling with $T_{c}$ is then equivalent to widening the harmonic potential when increasing the particle number, i.e. lowering the oscillation frequency towards zero (cf. eq. (3)).

We now address the question whether a macroscopic ground state population is a sufficient indicator to define Bose-Einstein condensation in this system. In this respect it is useful to consider another system with a constant density of states $\rho(E)$. From eq. (2) we find for a two-dimensional box of length $L$

$$
\rho(E)=\frac{m L^{2}}{2 \pi \hbar^{2}}
$$

Here a proper thermodynamic limit $N, L \rightarrow \infty$, with constant density $N / L^{2}$, can be performed and the notion of phase transition is well defined. While $\left(2 \pi \hbar^{2} / k_{\mathrm{B}} m\right)\left(N / L^{2}\right)$ provides the temperature scaling, either $N$ or $L^{2}$ determine how closely the thermodynamic limit is approached. 

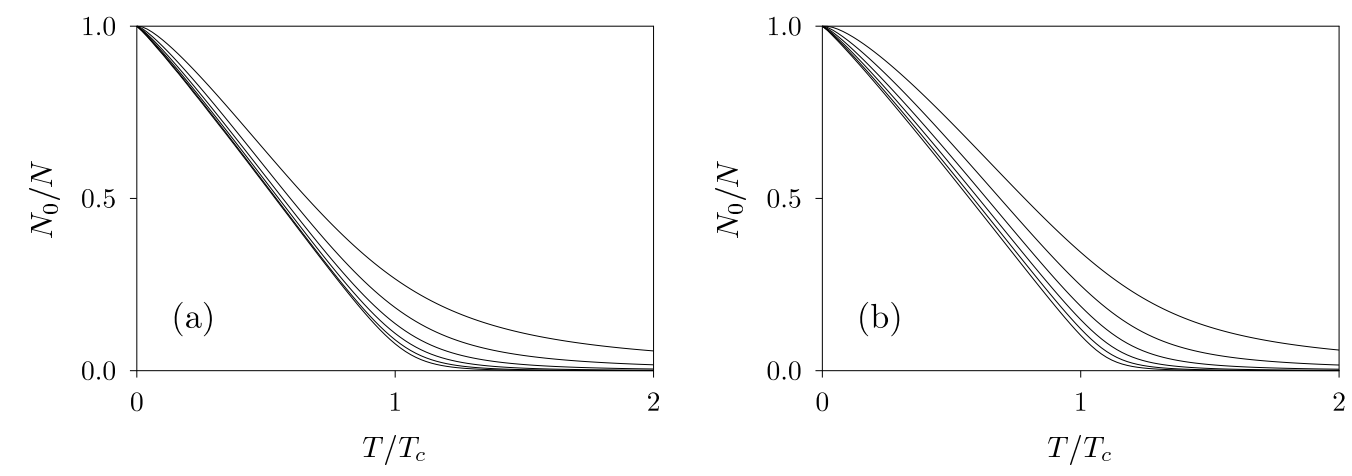

Fig. 1. - Relative ground state occupation $N_{0} / N$ (a) for the one-dimensional harmonic potential and (b) for the two-dimensional box as a function of temperature $T$ scaled with the critical temperature $T_{c}$. The numerical results obtained by taking into account the discrete level structure correspond to $N=10^{2}, 10^{3}, 10^{4}, 10^{5}, 10^{6}$, and $10^{7}$ particles from the upper right to the lower left curve.

For the two-dimensional box it is generally accepted that, in contrast to the three-dimensional box, no phase transition occurs (see [9], where the authors derive this conclusion, although the specific heat for the two-dimensional box is not correctly reproduced (14).

An analogy to the one-dimensional harmonic oscillator may be drawn by comparing its density of states $\rho(E)=1 / \hbar \omega$ to expression (4). The temperature scale is now given by $N \hbar \omega$ while the thermodynamic limit is reached either for $N \rightarrow \infty$ or $\hbar \omega \rightarrow 0$. For fixed frequency $\omega$ the limit $N \rightarrow \infty$ leads to a change in the temperature scale. A phase transition should therefore become apparent when rescaling the temperature with $N$. For later purposes we note that the dimensionless density for the two-dimensional box $N\left(\lambda_{\mathrm{T}} / L\right)^{2}$, where $\lambda_{\mathrm{T}}=\left(2 \pi \hbar^{2} \beta / m\right)^{1 / 2}$ is the thermal de Broglie wavelength, corresponds to $N \beta \hbar \omega$ for the one-dimensional harmonic oscillator.

To check the analogy between the two systems, we have calculated the ground state population for the two-dimensional box using discrete energy levels. Following the reasoning of Ketterle et al. [⿴囗玉 we define a critical temperature via $N=2\left(L / \lambda_{\mathrm{T}, \mathrm{c}}\right)^{2} \ln \left(L / \lambda_{\mathrm{T}, \mathrm{c}}\right)$ and obtain the results shown in fig. 1b. Clearly, as in the case of a one-dimensional harmonic potential, the ground state population for large $N$ shows a rather sharp transition to macroscopic values below the critical temperature. No qualitative difference in the behaviour of the two systems is visible. The definition of Bose-Einstein condensation via a macroscopic ground state population enters therefore in conflict with predictions for the two-dimensional box. For a good criterion for condensation one instead has to resort to thermodynamic quantities.

To this purpose we consider in particular the specific heat, which recently has become accessible experimentally 15

$$
C=\frac{\mathrm{d}}{\mathrm{d} T} \int_{0}^{\infty} \mathrm{d} E \rho(E) \frac{E z}{\exp (\beta E)-z} .
$$

Here $C$ is expressed through the continuous density of states $\rho(E)$, which will prove to be 
sufficient for our purposes. Evaluating (5) with constant density of states $\rho=1 / \hbar \omega$ yields

$$
\frac{C}{N k_{\mathrm{B}}}=\frac{2}{N \beta \hbar \omega} \sum_{n=1}^{\infty} \frac{z^{n}}{n^{2}}+\frac{1}{N \hbar \omega} \frac{\ln (1-z)}{z} \frac{\mathrm{d} z}{\mathrm{~d} \beta}
$$

and for the fugacity we obtain from (1)

$$
z=1-\exp (-\beta \hbar \omega N) .
$$

This expression is correct for high temperatures but breaks down when $z$ becomes of the order of its zero temperature value $N /(N+1)$, which is the case at temperatures of the order of the critical temperature defined by (3). At these temperatures $z$ almost takes the value of one and the difference to the approximation (7) is at most of order $1 / N$. Although (7) could not be used to determine the ground state population at low temperatures, it allows us to obtain the correct result for the specific heat. Corrections of order $1 / N$ to the fugacity are negligible in the first term on the right hand side of (6) whereas they are potentially dangerous in the logarithmic term. However in the temperature range of interest this term is strongly suppressed because $\mathrm{d} z / \mathrm{d} \beta$ becomes exponentially small.

With (7) the specific heat takes the form

$$
\frac{C}{N k_{B}}=\frac{2}{y} \sum_{n=1}^{\infty} \frac{(1-\exp (-y))^{n}}{n^{2}}-\frac{y}{\exp (y)-1}
$$

with $y=\beta \hbar \omega N$. The particle number $N$ enters this result only through a linear rescaling of the specific heat and the temperature. A similar result has been derived for a canonical ensemble 12. According to the above discussion the sum in (\$) determines the low temperature behaviour of the specific heat which is then found to be

$$
\frac{C}{N k_{B}}=\frac{\pi^{2}}{3} \frac{1}{y}=\frac{\pi^{2} k_{\mathrm{B}}}{3 N} \frac{1}{\hbar \omega} T .
$$

In view of the analogy between the one-dimensional harmonic oscillator and the two-dimensional box the specific heat of the latter one then becomes

$$
\left(\frac{C}{N k_{B}}\right)_{2 \mathrm{dbox}}=\frac{\pi^{2} k_{\mathrm{B}}}{3 N} \frac{m L^{2}}{2 \pi \hbar^{2}} T .
$$

In fig. 2, expression (8) is depicted by the full line with its low temperature approximation (9). The dashed line shows the result of a numerical calculation for 10 particles in a onedimensional harmonic potential taking into account the discreteness of the spectrum. Even for such small $N,(8)$ gives a rather good approximation. For 100 or more particles the difference between the result for the one-dimensional harmonic oscillator based on the discrete level structure on one hand and (8) on the other hand becomes negligible. For the two-dimensional box the convergence of the calculation using discrete energies to the result for a constant density of states is somewhat slower due to the higher dimensionality of the system.

Thus for large particle numbers the form of the specific heat becomes independent of $N$ and neither singularities build up in the thermodynamic limit $N \rightarrow \infty$ nor a maximum appears at the critical temperature like the one found in the result sketched in [9]. Furthermore, the limiting result (8) is analytic for finite temperatures and we conclude that, as in the two-dimensional box, there is no phase transition in a one-dimensional harmonic potential.

The difference between the behaviour of a non-interacting Bose gas in a one-dimensional and higher-dimensional harmonic potentials may also be understood in terms of discrete energy 


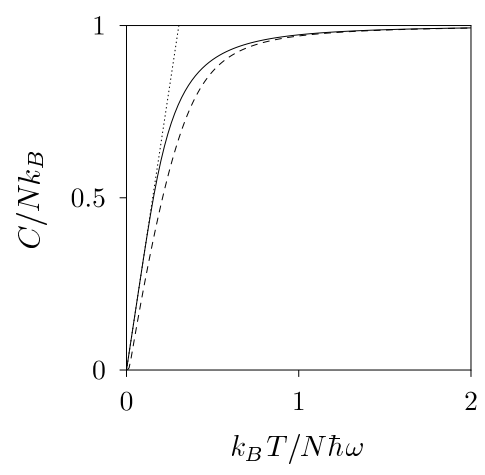

Fig. 2.

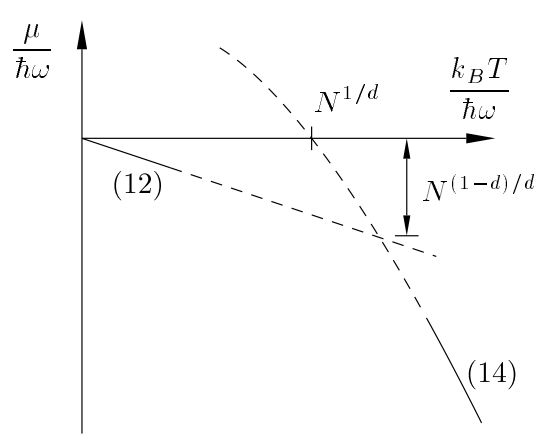

Fig. 3.

Fig. 2. - Specific heat $C$ of a Bose gas in a one-dimensional harmonic potential as a function of temperature $T$. The full line represents the result of a calculation with constant density of states with its low temperature approximation (dotted line), while the dashed line is for $N=10$ particles taking into account the discrete level structure. For $N=10$ eq. (3) yields $k_{B} T_{c} / N \hbar \omega=0.454$. Similar numerical results have been obtained in [12, 13.

Fig. 3. - Illustration of the reasoning leading to (15). The lines correspond to the upper bound of the chemical potential (12) and the high temperature approximation (14), respectively.

levels. An appropriate criterion for the possibility of Bose-Einstein condensation to occur is whether in the thermodynamic limit $N \rightarrow \infty$ the chemical potential equals the ground state energy below the critical temperature or not. In the following we will show that this criterion is not met in a one-dimensional harmonic potential. We start out with the average number of particles in a grand canonical description

$$
N=\frac{1}{\exp (-\beta \mu)-1}+\sum_{n=1}^{\infty} \frac{g_{n}^{(d)}}{\exp [\beta(\hbar \omega n-\mu)]-1},
$$

where $g_{n}^{(d)}$ is the degeneracy of the $n$-th eigenlevel of the $d$-dimensional harmonic potential. The first term on the right-hand-side of (11) represents the population of the ground state.

One derives an upper bound of the chemical potential at arbitrary temperatures by considering a situation where the excited states do not contribute to the number of particles

$$
\frac{\mu}{\hbar \omega} \leq-\frac{1}{N} \frac{k_{B} T}{\hbar \omega}
$$

Naturally this case coincides with the low temperature limit $\beta \hbar \omega \gg 1$. Chemical potential and temperature are taken with respect to the energy level spacing $\hbar \omega$ of the harmonic potential. This is the appropriate choice if $\omega$ is kept fixed in the thermodynamic limit.

For high temperatures the ground state population becomes very small and therefore $\beta|\mu| \gg 1$. We may then approximate expression (11) for the average particle number by

$$
N=\exp (\beta \mu) \sum_{n=0}^{\infty} g_{n}^{(d)} \exp (-\beta \hbar \omega n)=\frac{\exp (\beta \mu)}{(1-\exp (-\beta \hbar \omega))^{d}}
$$

Except for very low particle numbers the high temperature regime implies $\beta \hbar \omega \ll 1$. The 
chemical potential is then found as

$$
\frac{\mu}{\hbar \omega}=\frac{\ln \left[N(\beta \hbar \omega)^{d}\right]}{\beta \hbar \omega}
$$

and equals zero at $k_{B} T / \hbar \omega=N^{1 / d}$. Since the ground state energy was taken to be zero, the high temperature result suggests that there might be Bose-Einstein condensation below a temperature $\hbar \omega N^{1 / d} / k_{B}$. A more detailed analysis leads to a prefactor of the order of one.

To exclude the existence of a phase transition in a one-dimensional harmonic potential, it is sufficient to consider the upper bound (12) for the chemical potential at its intersection with the high temperature expression (14). There the value of the chemical potential becomes (cf. fig. 3)

$$
\frac{\mu}{\hbar \omega}=-N^{(1-d) / d}
$$

For $d=1$ this is of order one independently of the particle number $N$ and one expects a smooth crossover at the intersection point. This behaviour forbids a phase transition in the one-dimensional case. On the other hand, for $d \geq 2$, the chemical potential at the intersection vanishes for $N \rightarrow \infty$ opening the possibility of a sharp transition at a temperature of order $N^{1 / d}$, below which the chemical potential strictly equals the ground state energy. We thus recover the predictions of the theory based on the density of states (2).

In conclusion, by studying the specific heat and using an analogy with two-dimensional box systems we have shown that a macroscopic ground state population alone is not a sufficient indicator of a phase transition. In higher dimensional systems which show a condensation phenomenon a macroscopic ground state population is always accompanied by some signature in the specific heat or higher derivatives of the free energy. Their absence for the case of a one-dimensional harmonic potential leads us to conclude that no phase transition occurs in this system. The main interest of Bose-Einstein condensation lies in the particular coherence properties of condensates. Then the question arises to which extent the ground state population can give information about coherence. The results of this paper show that it might be necessary, maybe not sufficient, to carefully study thermodynamic quantities as well.

$$
* * *
$$

\section{REFERENCES}

[1] Anderson M. H., Ensher J. R., Matthews M. R., Wieman C. E. and Cornell E. A., Science, 269 (1995) 198.

[2] Bradley C. C., Sackett C. A., Tollett J. J. and Hulet R. G., Phys. Rev. Lett., 75 (1995) 1687.

[3] Davis K. B., Mewes M.-O., Andrews M. R., van Druten N. J., Durfee D. S., Kurn D. M. and Ketterle W., Phys. Rev. Lett., 75 (1995) 3969.

[4] Ketterle W. and van Druten N. J., Phys. Rev. A, 54 (1996) 656.

[5] London F., Phys. Rev., 54 (1938) 947.

[6] Bagnato V., Pritchard D. E. and Kleppner D., Phys. Rev. A, 35 (1987) 4354.

[7] Bagnato V. and Kleppner D., Phys. Rev. A, 44 (1991) 7439.

[8] Grossmann S. and Holthaus M., Z. Naturforsch., 50a (1995) 921.

[9] de Groot S. R., Hooyman G. J. and ten Seldam C. A., Proc. R. Soc. London Ser. A, 203 (1950) 266.

[10] Grossmann S. and Holthaus M., Z. Phys. B, 97 (1995) 319. 
[11] Kirsten K. and Toms D. J., Phys. Rev. A, 54 (1996) 4188.

[12] Brosens F., Devreese J. T. and Lemmens L. F., Solid St. Comm., 100 (1996) 123.

[13] Haugset T., Haugerud H. and Andersen J. O., Phys. Rev. A, 55 (1997) 2922.

[14] Ziff R. M., Uhlenbeck G. E. and Kac M., Phys. Rep., 32 (1977) 169.

[15] Ensher J. R., Jin D. S., Matthews M. R., Wieman C. E. and Cornell E. A., Phys. Rev. Lett., 77 (1996) 4984. 